\title{
A review of cemented tungsten carbide tool wear during wood cutting processes
}

\begin{abstract}
The aim of this review is to provide a complete state of the art review of the wear of cemented tungsten carbide tools during wood cutting processes. It examines the properties of cemented tungsten carbides and its wear mechanisms, under the influence of different working conditions. Further, it also elaborates the techniques that could be used to determine tool wear and also discusses the implications of tool wear.
\end{abstract}

Keyword: Cemented tungsten carbide; Tool wear; Wood; Cutting; Economics 